\title{
The role of PI3-K/Akt signal pathway in the antagonist effect of CEPO on CHF rats
}

\author{
ZHAOQI HUANG ${ }^{1}$, WEI XU ${ }^{2}$, JINLEI WU ${ }^{3}$, SHENGQIANG CHEN ${ }^{4}$ and XIMING CHEN ${ }^{1}$ \\ ${ }^{1}$ Department of Cardiovascular Medicine, The Third Affiliated Hospital of Guangzhou Medical University, \\ Guangzhou, Guangdong 510150; ${ }^{2}$ Department of Cardiovascular Medicine, Huadu District People's Hospital \\ of Guangzhou; ${ }^{3}$ Department of Cardiovascular Medicine, Hexian Memorial Affiliated Hospital of Southern \\ Medical University, Guangzhou, Guangdong 510000; ${ }^{4}$ Institute for Neurological Research, The Second \\ Affiliated Hospital of Guangzhou Medical University, Guangzhou, Guangdong 510260, P.R. China
}

Received March 8, 2018; Accepted September 11, 2018

DOI: $10.3892 /$ etm.2018.6822

\begin{abstract}
The possible role of phosphoinositide 3-kinase $(\mathrm{PI} 3-\mathrm{K}) /$ protein kinase $\mathrm{B}$ (Akt) signal pathway in the antagonist effect of carbamylated erythropoietin (CEPO) on chronic heart failure $(\mathrm{CHF})$ in rats was investigated. Twenty of 120 rats were randomly selected as the control group, and the remaining rats as the model group. Rats in the model group received intraperitoneal injection of isoproterenol, those in the control group underwent intraperitoneal injection of equivalent normal saline. Rats with successful model establishment were divided into 4 groups, i.e. CHF group, CEPO group, LY294002 (LY) group and CEPO + LY group. Rats in the CEPO group underwent intraperitoneal injection of $\mathrm{CEPO}$, while those in the $\mathrm{CHF}$ group received intraperitoneal injection of equivalent normal saline at the same time, those in the LY group received intraperitoneal injection of LY after model establishment, and those in the CEPO + LY group received the combined intraperitoneal injection of CEPO and LY simultaneously. Indicators for hemodynamics were determined using BL-410S bio-functional experiment system, including heart rate (HR), left ventricular end-diastolic pressure (LVEDP), left ventricular systolic pressure (LVSP) and maximal increased rate of left ventricular pressure $(\mathrm{LVP}) /$ maximal reduced rate of $\mathrm{LVP}\left( \pm \mathrm{dp} / \mathrm{dt}_{\max }\right)$. Western blotting assay was utilized to determine the changes in activity of PI3-K/Akt signal pathway. LVSP and $\pm \mathrm{dp} / \mathrm{dt}_{\max }$ in the CHF, the CEPO, the CEPO + LY and the LY groups were significantly lower than those in the control group $(\mathrm{P}<0.05)$; LVSP and $\pm \mathrm{dp} / \mathrm{dt}_{\max }$ in the CEPO group were also elevated significantly compared with CHF, LY and CEPO + LY
\end{abstract}

Correspondence to: Dr Ximing Chen, Department of Cardiovascular Medicine, The Third Affiliated Hospital of Guangzhou Medical University, 63 Duobao Road, Liwan, Guangzhou, Guangdong 510150, P.R. China

E-mail: 18078805442@163.com

Key words: CHF, CEPO, hemodynamics, PI3-K/Akt groups $(\mathrm{P}<0.05)$ with significant decreases in LVEDP and HR $(\mathrm{P}<0.05)$; compared with the CHF group, LVSP and $\pm \mathrm{dp} / \mathrm{dt}_{\max }$ in the LY group were each significantly decreased $(\mathrm{P}<0.05)$, in the LY group, pAkt level was significantly lower than that in the CHF group $(\mathrm{P}<0.05)$. In conclusion, $\mathrm{CEPO}$ can generate the antagonist effect on $\mathrm{CHF}$ in rats through activation of PI3-K/Akt signal pathway.

\section{Introduction}

With an aging population in the world, incidence rate of chronic heart failure (CHF) has been increasing year by year, resulting in an elevation in mortality rate (1). $\mathrm{CHF}$, as the end-stage of many cardiovascular diseases, refers to a clinical syndrome caused by organic or functional variations in the heart with major manifestations such as anomaly in heart structure, decrease in cardiac output caused by dysfunction of ventricular filling and/or ejection, persistent increase in venous pressure and gradual dysfunction in hemodynamics that can hardly satisfy the requirement of metabolism, which can result in progressive exacerbation in heart failure, necrosis in myocardial cells, thereby threatening the health and life quality of human beings; according to the degree of failure, $\mathrm{CHF}$ can be divided into three types: Left heart failure, right heart failure and whole heart failure (2). Besides, some younger patients may be the victims of $\mathrm{CHF}$, and, though $\mathrm{CHF}$ can be controlled by treatment, these patients are more susceptible to the recurrence of $\mathrm{CHF}$ for its irreversible and refractory features (3). Although inhibitor of renin-angiotensin system (RAS) is dominant in treatment of heart failure with the ability to stabilize or decrease the pressure and the protective effect on target organs, it brings about a variety of inevitable adverse reactions (4). Thus, searching for a new kind of drug with prominent efficacy and mild side effect is necessary.

In humans, erythropoietin (EPO), a glycoprotein secreted by kidneys, can bind to EPO receptor (EPOR) specifically, thereby activating the proliferation and differentiation of erythrocytes (5). Enormous number of studies have indicated that in addition to the function to activate the hematopoiesis, EPO manifests a protective activity on myocardial cells (6). EPO, through binding to EPOR, can activate EPOR to initiate 
multiple signal transduction pathways, like signal transducer and activator of transcription 5 (STAT5), phosphoinositide 3-kinase (PI3-K)/protein kinase B (Akt, or PKB) signal pathway, or mitogen-activated protein kinase (MAPK)/extracellular signal-regulated kinase (ERK) signal pathway, thereby protecting the tissues extensively (7). Not only can EPO protect the heart through increasing the oxygen supply to tissues by activating the generation of erythrocytes, but also it can exert its protective effect on the heart through multiple ways, including anti-apoptosis, anti-inflammation and proangiogenesis effects (8). Thereupon, medicinal EPO has been applied in treatment of relevant diseases, but, when applied as the protective agent, the dose of EPO should be higher than that in treatment of anemia (9), and such a high dose of EPO may give rise to dose-dependence, increased risks in coagulation and thrombosis, or even exacerbation in heart failure, which may frequently occur particularly in patients at high risk with thrombus, hypertension, polycythemia or hyperviscosity syndrome (10). Due to the adverse reactions above, application of EPO has been largely limited in clinical treatment of cardiovascular diseases. In 2004, Fiordaliso et al (11) in an in vitro experiment obtained carbamylated erythropoietin (CEPO), a derivative of EPO, which retains anti-inflammation, anti-apoptosis and tissue-protective effects that are similar to EPO but without pro-hematopoietic effect. CEPO is much safer than EPO in treatment of cardiovascular diseases with a much wider application prospect (11).

PI3-K/Akt (also called PKB) is a major signal pathway delivering the signal of anti-apoptosis/pro-proliferation, and PI3-K plays a key role in signal transduction pathway mediated by the growth factor receptor superfamily (12). Through binding to EPOR, EPO can activate PI3-K that will further catalyze the transformation of diphosphoinositide (PIP2) into triphosphoinositide (PIP3), which, as a second messenger, can activate the following multiple target proteins, thereby regulating the proliferation, differentiation, migration and transplantation of cells (13). Akt, also called serine/threonine protein kinase $\mathrm{B}(\mathrm{PKB})$, is one of the downstream target proteins of PI3-K; once activated, Akt can be phosphorylated into phosphorylated Akt (pAkt), which can further phosphorylate a series of apoptotic regulation factors of $\mathrm{B}$-cell lymphoma-2 (Bcl-2)/ B-cell lymphoma-extra-large (Bcl-XL)associated death promoter (BAD), caspase and nuclear factor $(\mathrm{NF})-\kappa \mathrm{B}$, so as to affect the transcription of anti-apoptosis genes, which is conducive to survival of cells (14). In this study, we investigated whether CEPO could antagonize CHF in rats through the PI3-K/Akt signal pathway.

\section{Materials and methods}

\section{Materials}

Experimental animals. A total of 120 healthy clean male Wistar rats weighing 200-240 g were purchased from Experimental Animal Center of Academy of Military Medical Sciences (Beijing, China) [approval no. SCXK-(Military) 2012 0004] and fed with regular food in separate cages at $20-25^{\circ} \mathrm{C}$ and $60-70 \%$ humidity-controlled environment, in which rats had free access to water that was disinfected through ultraviolet. Adjustment in light and ventilation was performed in accordance with the standards. Padding was changed twice per week, and regular food was given in $25 \mathrm{~g} / \mathrm{rat} / \mathrm{day}$. Experiment was carried out following several days of acclimatization. The study was approved by the Ethics Committee of The Third Affiliated Hospital of Guangzhou Medical University (Guangzhou, China).

Reagents. Before administration of CEPO (Amgen, Thousand Oaks, CA, USA), the dose of CEPO was calculated and weighed in $50 \mu \mathrm{g} / \mathrm{kg}$ with reference of the total weight of rats in CEPO group. Then CEPO was dissolved in double distilled water until well mixed, and the concentration of CEPO was adjusted to $100 \mu \mathrm{g} / \mathrm{ml}$; isoproterenol (ISO; $2 \mathrm{ml}, 1 \mathrm{mg} /$ bottle, Shanghai Harvest Pharmaceutical Co. Ltd., Shanghai, China); LY294002 (LY) (Selleck Chemicals, Houston, TX, USA); enhanced chemiluminescence (ECL) kit (Santa Cruz Biotechnology, Inc., Dallas, TX, USA); Rabbit anti-rat pAkt (Ser473) and Akt monoclonal antibodies (cat. nos. 4058 and 4685 respectively; Cell Signaling Technology, Inc., Danvers, MA, USA); horseradish peroxidase labeled anti-rabbit IgG polyclonal antibody (cat. no. 7074; Cell Signaling Technology, Inc.). Bicinchoninic acid (BCA) protein assay reagent kit (Pierce; Thermo Fisher Scientific, Inc., Waltham, MA, USA).

\section{Methods}

Establishment of CHF models in rats. After being fed for several days to acclimatize to the environment, 20 of the rats were selected as the control group, and the remaining rats as the model group, where rats underwent intraperitoneal injection of ISO $(5 \mathrm{mg} / \mathrm{kg})$ once per day for 3 days to establish the models. At the same time, rats in the control group underwent intraperitoneal injection of equivalent normal saline. During intraperitoneal injection, needle should be inserted into the abdomen. Pumped back to see whether the blood or intestinal fluid was drawn, so as to avoid the drugs being delivered into the vessels, which could lead to the death of rats, or into the bladder or intestinal tube to lose the activity of drugs. After 5 weeks of feeding, survived rats were delivered to evaluate the model establishment. During the feeding period, a total of 8 rats in the model group died, which might have been caused by the rapid injection of ISO or delivery of drugs to vessels due to inappropriate operations, leading to embolism, or any other unknown factors. In examination of hemodynamics, left ventricular end-diastolic pressure (LVEDP) $\geq 15 \mathrm{mmHg}$ suggested that models were established successfully (15).

Grouping of animals. After the hemodynamics examination for identifying the successful model establishment, wounds in rats were sutured followed by administration of gentamycin at a dose of $24,000 \mathrm{U} / \mathrm{kg} /$ day for 3 days to prevent infections. A total of 92 rats with successful model establishment were divided into 4 groups randomly, i.e. CHF group $(n=23)$, CEPO group $(n=23)$, LY group $(n=23)$ and CEPO + LY group $(n=23)$.

CEPO group: After being weighed, rats in CEPO group underwent intraperitoneal injection of CEPO at a dose of $50 \mu \mathrm{g} / \mathrm{kg}$ for 4 weeks (16).

$\mathrm{CHF}$ group and control group: Rats in $\mathrm{CHF}$ group received intraperitoneal injection of equivalent normal saline at the same time.

The rats in LY group, after being weighed, received intraperitoneal injection of LY at a dose of $0.25 \mu \mathrm{g} / 100 \mathrm{~g}$ twice per week for 4 weeks. 
Table I. Comparison of the hemodynamics indicators among groups (mean \pm SD).

\begin{tabular}{lccccc}
\hline & Control group & CHF group & CEPO group & CEPO+LY group & LY group \\
\hline $\mathrm{n}$ & 20 & 23 & 23 & 23 & 23 \\
$\mathrm{LVSP}(\mathrm{mmHg})$ & $134.71 \pm 6.31$ & $108.40 \pm 8.24^{\mathrm{a}}$ & $119.72 \pm 7.84^{\mathrm{ab}}$ & $112.48 \pm 8.01^{\mathrm{ac}}$ & $97.2 \pm 6.76^{\mathrm{a}-\mathrm{c}}$ \\
$+\mathrm{dp} / \mathrm{dt}_{\max }(\mathrm{mmHg} / \mathrm{sec})$ & $4444.54 \pm 230.56$ & $2965.74 \pm 235.41^{\mathrm{a}}$ & $3721.50 \pm 200.07^{\mathrm{ab}}$ & $3328.64 \pm 210.43^{\mathrm{ac}}$ & $2794.47 \pm 198.52^{\mathrm{a}-\mathrm{c}}$ \\
$-\mathrm{dp} / \mathrm{dt}_{\max }(\mathrm{mmHg} / \mathrm{sec})$ & $3926.60 \pm 208.78$ & $2879.95 \pm 219.93^{\mathrm{a}}$ & $3343.28 \pm 207.41^{\mathrm{ab}}$ & $3014.7 \pm 209.87^{\mathrm{ac}}$ & $2689.98 \pm 207.34^{\mathrm{a}-\mathrm{c}}$ \\
$\mathrm{LEVDP}(\mathrm{mmHg})$ & $5.36 \pm 0.78$ & $19.46 \pm 1.17^{\mathrm{a}}$ & $11.70 \pm 1.14^{\mathrm{ab}}$ & $14.43 \pm 1.07^{\mathrm{ac}}$ & $22.62 \pm 1.21^{\mathrm{a}-\mathrm{c}}$ \\
$\mathrm{HR}($ beat $/ \mathrm{min})$ & $340.43 \pm 11.61$ & $416.11 \pm 13.63^{\mathrm{a}}$ & $388.84 \pm 11.85^{\mathrm{ab}}$ & $427.84 \pm 12.43^{\mathrm{ac}}$ & $470.46 \pm 11.93^{\mathrm{a}-\mathrm{c}}$ \\
\hline
\end{tabular}

${ }^{\mathrm{a}} \mathrm{P}<0.05$ in comparison with control group; ${ }^{\mathrm{b}} \mathrm{P}<0.05$ in comparison with $\mathrm{CHF}$ group; ${ }^{\mathrm{c}} \mathrm{P}<0.05$ in comparison with $\mathrm{CEPO}$ group. $\mathrm{CHF}$, chronic heart failure; CEPO, carbamylated erythropoietin; LY, LY294002; LVSP, left ventricular systolic pressure; LEVDP, left ventricular end-diastolic pressure; HR, heart rate.

Rats in CEPO + LY group, after being weighed, received intraperitoneal injection of LY at a dose of $0.25 \mu \mathrm{g} / 100 \mathrm{~g}$ firstly for pretreatment followed by intraperitoneal injection of CEPO at a dose of $50 \mu \mathrm{g} / \mathrm{kg}$ twice per week for 4 weeks.

After 4 weeks, indicators for hemodynamics were determined in all survived rats, including heart rate (HR), LVEDP, left ventricular systolic pressure (LVSP) and maximal increased rate of left ventricular pressure (LVP)/maximal decreased rate of $\operatorname{LVP}\left( \pm \mathrm{dp} / \mathrm{dt}_{\max }\right)$. Western blotting assay was utilized to determine the changes in activity of PI3-K/Akt signal pathway

Examination of hemodynamics. After $12 \mathrm{~h}$ of fasting of both food and water, rats were anesthetized using urethane $(25 \%$, $300 \mathrm{mg} / \mathrm{kg}$ ), and fixed in a plate in supine position. Following the regular skin preparation and disinfection, skin of neck was incised to separate the right common carotid artery (CCA) with a $2-\mathrm{cm}$ segment being freed and exposed. The distal end of CCA was ligated, and the proximal end was clamped with a small bulldog clamp. About $1.5 \mathrm{~cm}$ in front of the ligature, polyethylene catheter (containing $0.1 \%$ heparin-normal saline; diameter of $1 \mathrm{~mm}$; micro-pressure sensor deployed at the end of catheter) of BL-410S bio-function experiment system (Beijing Temo Technology Co., Ltd., Beijing, China) was inserted through puncture. Then, the bulldog clamp was open, and catheter was guided into the left ventricle through ascending aorta with smooth forceps until the significant flat peak wave in diastolic phase of pressure signal on the screen, indicating that catheter was delivered into the left ventricle. After 10 min of stabilization, indicators were recorded.

Detection of pAkt and Akt through western blotting assay. From each group, 4 animals were taken for treatment, and heart tissues were dissected and shifted into the liquid $\mathrm{N}_{2}$ within $24 \mathrm{~h}$ for preservation and later use. In accordance with the regular method, total proteins in heart tissues were extracted for protein quantification with BCA protein quantification kit. Gel was prepared with $10 \%$ separation gel and $3 \%$ spacer gel, and proteins in cytoplasm were extracted in the mixture of 6X sodium dodecyl sulfate (SDS, 2\%) and sample buffer. Five minutes after the temperature reached $95^{\circ} \mathrm{C}, 10 \mu \mathrm{l}$ samples were loaded for electrophoresis until the bromophenol blue gathered at the bottom of separation gel. Polyvinylidene fluoride membrane was immersed in membrane-transfer buffer for $15 \mathrm{~min}$, and semi-dry membrane transfer was then initiated with gel in contact with the negative electrode of semi-dry transfer cell membrane-transfer apparatus (Bio-Rad Laboratories, Inc., Hercules, CA, USA) and PVDF with the positive electrode under a constant voltage of $15 \mathrm{~V}$ for 2-3 $\mathrm{h}$. Thereafter, membrane was blocked in $4^{\circ} \mathrm{C}$ blocking buffer [5\% skimmed milk powder and $1 \mathrm{X}$ Tris-buffered salineTween-20 (TBS-T)] overnight. Membrane was then incubated with primary antibody (Akt, 1:400; pAkt, 1:400) overnight at $4^{\circ} \mathrm{C}$, followed by washing. After that, horseradish peroxidase labeled anti-rabbit $\operatorname{IgG}(1: 2,000)$ was added on the membrane which was later prepared for color development reaction using chemiluminescent method with ECL kit. The X-ray images were prepared for color development. Scanning and identification of images: In this study, the quantitative analysis of grey value was performed with MHImage 1.63 image analysis system.

Statistical analysis. With Statistical Product and Service Solutions (SPSS 21.0; IBM Corp., Armonk, NY, USA), t-test and one-way analysis of variance (one-way ANOVA) were performed and SNK test was the post hoc test. Measurement data are presented as mean \pm standard deviation (SD). $\mathrm{P}<0.05$ was set as the critical value.

\section{Results}

Observation of general manifestations. During the whole test, manifestations in the CHF group such as dry and shaggy hair without gloss, lack of energy, drowsiness and hypoactivity, and decline in food were observed sequentially, and in some severe cases, manifestations including massive loss of hair, disturbance in respiration, cyanosis and persistent poor sense on balance. Symptoms of heart failure in varying degrees were also observed in rats of the CEPO group before injection of CEPO, but were ameliorated significantly after medication. However, in the CEPO + LY group, after medication, symptoms of heart failure in varying degrees that were observed before medication had no significant improvement. In addition, symptoms of heart failure of rats in the LY group were more severe than those in the CHF group.

Hemodynamics indicators. As shown in Table I, LVSP and $\pm \mathrm{dp} / \mathrm{dt}_{\max }$ in the CHF, CEPO, CEPO + LY and LY groups were 


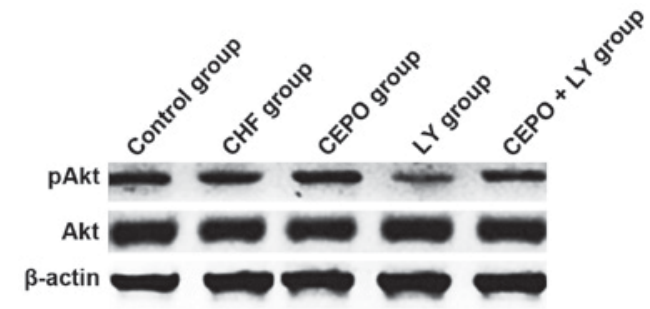

Figure 1. Expression of Akt and pAkt in rats of all groups. Expression of Akt and pAkt in rats of all groups through western blotting assay. Akt, protein kinase B; pAkt, phosphorylated Akt; CHF, chronic heart failure; CEPO, carbamylated erythropoietin; LY, LY294002.

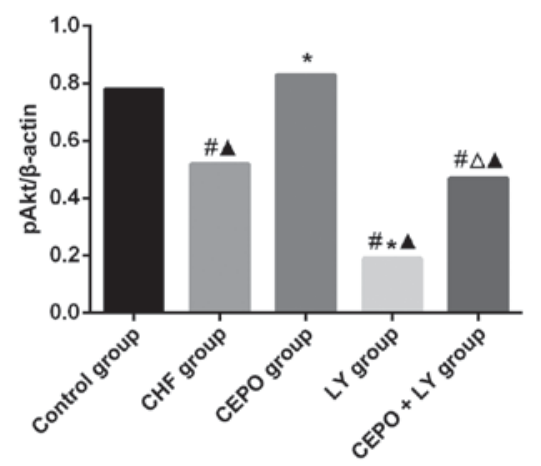

Figure 2. Statistical diagram of relative expression of pAkt. ${ }^{~} \mathrm{P}<0.05$ in comparison with the CEPO group; ${ }^{\wedge} \mathrm{P}<0.05$ in comparison with the LY group; ${ }^{*} \mathrm{P}<0.05$ in comparison with the CHF group; ${ }^{\wedge} \mathrm{P}<0.05$ in comparison with the control group. Akt, protein kinase B; pAkt, phosphorylated Akt; CHF, chronic heart failure; CEPO, carbamylated erythropoietin; LY, LY294002.

significantly lower than those in the control group $(\mathrm{P}<0.05)$, while LVEDP and HR in these groups were increased obviously when compared with the control group $(\mathrm{P}<0.05)$; in comparison with the CHF, LY and CEPO + LY groups, LVSP and $\pm \mathrm{dp} / \mathrm{dt}_{\max }$ were elevated significantly in the CEPO group $(\mathrm{P}<0.05)$ with significant decrease in LVEDP and HR $(\mathrm{P}<0.05)$; when compared with the CHF group, LVSP and $\pm \mathrm{dp} / \mathrm{dt}_{\max }$ in the LY group were significantly decreased $(\mathrm{P}<0.05)$ with remarkable elevations in LVEDP and HR $(\mathrm{P}<0.05)$.

Comparison of results in western blotting assay. In comparison with the control group, pAkt levels in CHF, CEPO + LY and LY groups were significantly declined $(\mathrm{P}<0.05)$, while that in the CEPO group was significantly higher $(\mathrm{P}<0.05)$; in the LY group, pAkt level was significantly lower than that in the CHF group $(\mathrm{P}<0.05)$, suggesting that $\mathrm{CEPO}$ increased the phosphorylation level of Akt, which was inhibited by LY. Comparisons of Akt levels among groups showed that differences had no statistical significance (Figs. 1 and 2).

\section{Discussion}

CHF is one of the major causes of death contributed by cardiovascular diseases. Once the cardiac output is decreased to a degree that can hardly satisfy the requirement of the metabolism, a series of organic or functional variations take place, which can threaten the safety and quality of life of humans (17). CEPO, as a carbamylated derivative of EPO, has protective effect on myocardial cells but no pro-hematopoietic effect (18). Millet et al (19) reported that in the nerve system, EPO and CEPO can inhibit the opening of mitochondrial permeability transition pore (mPTP) to sustain the membrane potential of mitochondria and calcium homeostasis, thereby reducing the death of cells induced by ischemia. Similarly, other researchers $(20,21)$ also confirmed that EPO and CEPO have nourishing and protective effect on nerves.

LVSP mainly reflects the systolic function of myocardium, and decrease of LVSP suggests a decline in systolic function of myocardium; $+\mathrm{dp} / \mathrm{dt}_{\max }$ can also indicate the performance of the myocardium in systolic phase almost regardless of the effect of load, and its decrease means that the systolic capability of myocardium is decreased; - $\mathrm{dp} / \mathrm{dt}_{\max }$ serves as an indicator of diastolic capability of myocardium, and its decrease reflects that the diastolic capability of myocardium is curbed; LVEDP is used to evaluate the preload of left ventricle and reflect the diastolic capability of myocardium, and the increase of LVEDP shows that the diastolic capability of myocardium is weakened (22). In this study, diastolic and systolic capabilities of rats in CHF group were decreased, which, however, were ameliorated after medication of CEPO. In addition, pretreatment of LY blocked the ability of CEPO to ameliorate the heart function. Results of western blotting assay showed that CEPO could elevate the level of pAkt, which was inhibited by treatment of LY, suggesting that CEPO can ameliorate the heart function of CHF rats through PI3-K/Akt signal pathway. Results in this study agreed with those of He et al (23).

In addition to the prophylactic effect on apoptosis of myocardial cells and protective effect on myocardial cells, normal activation of PI3-K/Akt signal pathway is also indispensable to other life activities (2,24-28). However, excessive activation of this signal pathway may promote the development of cancer (29). In this study, LVSP and $\pm \mathrm{dp}^{-\mathrm{dt}_{\max }}$ were decreased and LVEDP and HR increased in the LY group compared with in the CHF group, which might be caused by the blocking effect of LY on PI3-K/Akt signal pathway; this further affected the life activities associated with this signal pathway, and the descended life quality could hardly be sustained, which exacerbated the CHF in rats, further weakening the diastolic and systolic capabilities of the heart.

In conclusion, CEPO can generate the antagonist effect on $\mathrm{CHF}$ in rats through activation of PI3-K/Akt signal pathway.

\section{Acknowledgements}

Not applicable.

\section{Funding}

This study was funded by The Department of Science and Technology of Guangdong Province (project no. 2013B022000103).

\section{Availability of data and materials}

The datasets used and/or analyzed during the present study are available from the corresponding author on reasonable request. 


\section{Authors' contributions}

$\mathrm{ZH}$ contributed significantly to writing the manuscript and establishment of CHF models. WX and JW helped with animal grouping and treatment. SC conducted examination of hemodynamics. XC performed western blot analysis. All authors read and approved the final manuscript.

\section{Ethics approval and consent to participate}

The study was approved by the Ethics Committee of The Third Affiliated Hospital of Guangzhou Medical University (Guangzhou, China).

\section{Patient consent for publication}

Not applicable.

\section{Competing interests}

The authors declare that they have no competing interests.

\section{References}

1. Ismailov RM: Global heart failure rates and erythropoietin. Iran J Med Hypotheses Ideas 6: 70-74, 2012.

2. Zhang XJ, Liu J, Wan L, Sun Y, Wang F, Qi YJ and Huang CB: Relations of synovial angiogenesis and PTEN/PI3K/AKT signaling pathway in rats with adjuvant arthritis. Zhongguo $\mathrm{Gu}$ Shang 28: 71-74, 2015 (In Chinese).

3. Polat N, Oz F, Baykız D, Cizgici AY, Altun I, Buğra Z, Umman B, Tufan $\mathrm{F}$ and Oflaz H: Predictors of functional capacity in younger and elderly chronic heart failure patients: An observational study. Anadolu Kardiyol Derg 13: 778-783, 2013.

4. Iwanami J, Mogi M, Iwai M and Horiuchi M: Inhibition of the renin-angiotensin system and target organ protection. Hypertens Res 32: 229-237, 2009.

5. Suzuki N, Mukai HY and Yamamoto M: In vivo regulation of erythropoiesis by chemically inducible dimerization of the erythropoietin receptor intracellular domain. PLoS One 10 e0119442, 2015

6. Wan LJ and Wu XH: Effects of erythropoietin on cardiomyocyte apoptosis and endoplasmic reticulum stress-related proteins in neonatal rats with asphyxia. Zhongguo Dang Dai Er Ke Za Zhi 15: 890-895, 2013 (In Chinese).

7. Chang ZY, Yeh MK, Chiang $\mathrm{CH}$, Chen $\mathrm{YH}$ and Lu DW: Erythropoietin protects adult retinal ganglion cells against NMDA-, trophic factor withdrawal-, and TNF- $\alpha$-induced damage. PLoS One 8: e55291, 2013.

8. Doue T, Ohtsuki K, Ogawa K, Ueda M, Azuma A, Saji H, Strauss HW and Matsubara H: Cardioprotective effects of erythropoietin in rats subjected to ischemia-reperfusion injury: Assessment of infarct size with $99 \mathrm{mTc}$-annexin V. J Nucl Med 49: 1694-1700, 2008.

9. Wen Y, Xu J, Ma X and Gao Q: High-dose erythropoietin in acute ST-segment elevation myocardial infarction: A meta-analysis of randomized controlled trials. Am J Cardiovasc Drugs 13 435-442, 2013.

10. Minamino T, Toba K, Higo S, Nakatani D, Hikoso S, Umegaki M, Yamamoto K, Sawa Y, Aizawa Y and Komuro I; EPO-AMI-II study investigators: Design and rationale of low-dose erythropoietin in patients with ST-segment elevation myocardial infarction (EPO-AMI-II study): A randomized controlled clinical trial. Cardiovasc Drugs Ther 26: 409-416, 2012.

11. Fiordaliso F, Chimenti S, Staszewsky L, Bai A, Carlo E, Cuccovillo I, Doni M, Mengozzi M, Tonelli R, Ghezzi P, et al: A nonerythropoietic derivative of erythropoietin protects the myocardium from ischemia-reperfusion injury. Proc Natl Acad Sci USA 102: 2046-2051, 2005.
12. Kretz A, Happold CJ, Marticke JK and Isenmann S: Erythropoietin promotes regeneration of adult CNS neurons via Jak2/Stat3 and PI3K/AKT pathway activation. Mol Cell Neurosci 29: 569-579, 2005.

13. Lu X, Lv S, Mi Y, Wang L and Wang G: Neuroprotective effect of miR-665 against sevoflurane anesthesia-induced cognitive dysfunction in rats through PI3K/Akt signaling pathway by targeting insulin-likegrowthfactor2.AmJTranslRes9:1344-1356, 2017.

14. Gasparotto EPL, Tognon R, Ferreira AF, Oliveira GLV, Palma PVB, Zanichelli MA, Souto EX, Velano CEE, Carrara RCV, Kashima S, et al: Deregulated expression of Al, Bcl-2, Bcl-xL, and Mcl-1 antiapoptotic proteins and Bid, Bad, and Bax proapoptotic genes in polycythemia vera patients. Braz J Pharm Sci 47: 873-886, 2011.

15. Sattarzadeh R, Tavoosi A and Tajik P: Echocardiographic estimation of left ventricular filling pressures in patients with mitral valve stenosis. Cardiovasc J Afr 25: 34-39, 2014

16. Li Y, Takemura G, Okada H, Miyata S, Maruyama R, Li L, Higuchi M, Minatoguchi S, Fujiwara T and Fujiwara $\mathrm{H}$ : Reduction of inflammatory cytokine expression and oxidative damage by erythropoietin in chronic heart failure. Cardiovasc Res 71: 684-694, 2006.

17. Martins QC, Aliti G and Rabelo ER: Decreased cardiac output: Clinical validation in patients with decompensated heart failure. Int J Nurs Terminol Classif 21: 156-165, 2010.

18. Liu W, Shen Y, Plane JM, Pleasure DE and Deng W: Neuroprotective potential of erythropoietin and its derivative carbamylated erythropoietin in periventricular leukomalacia. Exp Neurol 230: 227-239, 2011.

19. Millet A, Bouzat P, Trouve-Buisson T, Batandier C, PernetGallay K, Gaide-Chevronnay L, Barbier EL, Debillon T, Fontaine E and Payen JF: Erythropoietin and its derivates modulate mitochondrial dysfunction after diffuse traumatic brain injury. J Neurotrauma 33: 1625-1633, 2016.

20. Li X, Bai ZY, Zhang FY and Xu XY: Neuroprotection of herbs promoting EPO on cerebral ischemia. Zhongguo Zhong Yao Za Zhi 40: 2265-2271, 2015 (In Chinese).

21. Gu YX, Du J, Si MS, Mo JJ, Qiao SC and Lai HC: The roles of PI3K/Akt signaling pathway in regulating MC3T3-E1 preosteoblast proliferation and differentiation on SLA and SLActive titanium surfaces. J Biomed Mater Res A 101: 748-754, 2013.

22. Stewart JT, Grbic M and Sigwart U: Left atrial and left ventricular diastolic function during acute myocardial ischaemia. Br Heart J 68: 377-381, 1992.

23. He H, Qiao X and Wu S: Carbamylated erythropoietin attenuates cardiomyopathy via PI3K/Akt activation in rats with diabetic cardiomyopathy. Exp Ther Med 6: 567-573, 2013.

24. Ueba H, Brines M, Yamin M, Umemoto T, Ako J, Momomura S, Cerami A and Kawakami M: Cardioprotection by a nonerythropoietic, tissue-protective peptide mimicking the 3D structure of erythropoietin. Proc Natl Acad Sci USA 107: 14357-14362, 2010.

25. Chen Q, Xu T, Li D, Pan D, Wu P, Luo Y, Ma Y and Liu Y: JNK/ $\mathrm{PI} 3 \mathrm{~K} / \mathrm{Akt}$ signaling pathway is involved in myocardial ischemia/ reperfusion injury in diabetic rats: effects of salvianolic acid A intervention. Am J Transl Res 8: 2534-2548, 2016.

26. de Oliveira MR, Ferreira GC, Schuck PF and Dal Bosco SM: Role for the PI3K/Akt/Nrf2 signaling pathway in the protective effects of carnosic acid against methylglyoxal-induced neurotoxicity in SH-SY5Y neuroblastoma cells. Chem Biol Interact 242: 396-406, 2015.

27. Wang N, Li T and Han P: The effect of tianmai xiaoke pian on insulin resistance through PI3-K/AKT signal pathway. J Diabetes Res 2016: 9261259, 2016.

28. Cen L, Hsieh FC, Lin HJ, Chen CS, Qualman SJ and Lin J: PDK-1/AKT pathway as a novel therapeutic target in rhabdomyosarcoma cells using OSU-03012 compound. Br J Cancer 97: 785-791, 2007.

29. Wei X, Wang G, Li W, Hu X, Huang Q, Xu K, Lou W, Wu J, Liang C, Lou Q, et al: Activation of the JAK-STAT3 pathway is associated with the growth of colorectal carcinoma cells. Oncol Rep 31: 335-341, 2014

This work is licensed under a Creative Commons Attribution-NonCommercial-NoDerivatives 4.0 International (CC BY-NC-ND 4.0) License. 